\title{
LOS SISTEMAS INTERNACIONALES NO EUROPEOS. RESEÑA DEL LIBRO: RINGMAR, E. (2019). HISTORY OF INTERNATIONAL RELATIONS: A NON-EUROPEAN PERSPECTIVE. OPEN BOOK PUBLISHERS.
}

\author{
Tatiana Peña Sequeira \\ ORCID: 0000-0002-0411-1985
}

La disciplina académica de Relaciones Internacionales ha sido criticada sistemáticamente por su eurocentrismo. En este marco, el libro History of International Relations: A Non-European Perspective, publicado en 2019, tiene una gran importancia pues busca abordar esa falta de profundidad en la historia de las Relaciones Internacionales, pues se ha concentrado en el imperialismo europeo dejando de lado gran parte de la experiencia de la humanidad.

Su autor, Erik Ringmar de Suecia, se graduó de doctorado en 1993 en la Universidad de Yale, y se desempeña en la actualidad como profesor en el Departamento de Ciencias Políticas y Relaciones Internacionales de la Universidad Ibn Haldun en Estambul, Turquía. Ringmar considera que para entender la historia del mundo y cómo funciona en la actualidad, la versión europea ya no es suficiente, por lo que es necesario volver a las historias de otras civilizaciones. A través de una revisión histórica desde el primer milenio de la "Era Común", hace un estudio comparativo de los sistemas internacionales previos al europeo, específicamente de China y Asia del Este, India, los califatos musulmanes, los kanatos mongoles, África y las Américas.

El autor define que un sistema está compuesto por unidades que trabajan de manera autónoma, que comparten el mismo medio, el cual influye en cómo se comportan; es por esto el comportamiento de una de estas unidades también depende del comportamiento de las demás, habiendo un efecto del sistema que se mide en términos de la interacción (Ringmar, 2019). En cuanto al sistema internacional, este está hecho de unidades políticas, los Estados, que trabajan de manera separada pero que se comportan en relación con las otras unidades 
del sistema, determina qué se puede hacer y que no, porque tiene instituciones, normas y reglas que lo definen y que, dependiendo del sistema, se les va a dar más énfasis que en otros. Además, como lo explica Ringmar (2019), se va a utilizar al Estado como el sujeto de la historia, argumenta que la historia del mundo es igual a la historia del Estado, siendo esto una limitación al determinar que civilizaciones tuvieron un sistema y las que no.

Para explicar la forma en la que la civilización se expande, el autor compara la cultura con la agricultura, porque para proteger y cuidar lo que se cosecha se crean barreras, así sucede con las culturas, los límites protegen las forma de vida para que estas no cambien y se mantengan. Tal es el ejemplo de ciertos grupos que no tuvieron gran interacción con el resto del mundo, que los hizo autosuficientes e independientes, porque se aislaron de influencias externas que le provocaran el cambio. Es por esto que el autor considera que si la cultura se puede comparar con la agricultura, la civilización lo hace con el intercambio, porque argumenta que la civilización depende del flujo de bienes, ideas, creencias y formas de vida, y que cuando se les da a las personas diferentes opciones, ya sea con sus bienes o de una manera más profunda, con sus costumbres; estos tomaran la opción de cambiar, y que es ahí cuando la civilización transforma la cultura y esto es lo que permite que civilización se expanda, aunque a costa de la cultura. Sin embargo, esta explicación justifica el expansionismo europeo, aunque argumenta que lo que los europeos hicieron fue adverso, también estima que conllevó beneficios, y que se va a tratar de un tema "controversial".

Para Ringmar, en el siglo XXI solo hay un sistema internacional, el europeo, y que este se debe la historia de imperialismo y colonialismo que permitió la expansión a todas las partes del mundo de diversas maneras (Ringmar, 2019). Entre estas, la conquista de América, el Norte de África, y de partes de Asia; el establecimiento de la Compañía Neerlandesa de las Indias Orientales (la Vereenigde Oostindische Compagnie) y de la Compañía Británica de las Indias Orientales (el East India Company); también el comercio con China y Japón, después de obligarlos a abrir sus mercados, así como la división de África en la Conferencia de Berlín (Ringmar, 2019). Todo esto conllevó, primero, que los europeos se sintiesen que tenían el derecho de mandar por encima de los demás, pero también creó un mundo que era solo aceptado si estaba hecho a su imagen y semejanza, con sus mismas reglas, normas e instituciones, lo que resultó en un solo sistema internacional (Ringmar, 2019). Sin embargo, explica que, previo a estas circunstancias, existían otros tipos de sistemas internacionales alrededor del mundo.

En específico, en China, Japón, India, los califatos musulmanes, y los kanatos mongoles había un sistema internacional. En el caso de China, el autor determina 
dos momentos en los que se puede identificar un sistema internacional, en la época de los "reinos combatientes", que, al dividirse en siete estados, se volvió por su mismo un sistema internacional. Posteriormente Qin Shing Huan, unificó los estados y China se convirtió en el país dominante en el Este de Asia, como centro de un sistema internacional. Estos tenían un sistema de tributos en que los demás estados se sometían a las ceremonias y los requerimientos del emperador para comerciar con China, se dice que, en la dinastía Ming, 123 países participaban de estas ceremonias.

En Japón, durante el periodo de Tokugawa cuando este se encontraba cerrado al mundo exterior y en donde su gobierno era el centro, aunque no tenía control sobre todo el territorio, sí tenían reuniones con los líderes de las 250 regiones que lo componían, lo que lo hace un mini sistema internacional. En el caso de India, cuando el subcontinente estaba unido, en ese momento Bangladesh, Pakistán y Sri Lanka, el autor lo determina como un sistema internacional dentro de sí mismo que se mantenía integrado por la identidad que comparten, en cuando a sus prácticas y ceremonias hindúes. En cuanto a los califatos musulmanes, el autor lo determina como un sistema internacional de los califatos, que se comportaban como una federación que se mantenía unido por el lenguaje y la religión que compartían.

Por último, determina que en los kanatos mongoles había un sistema internacional cuando se divide el imperio en la Horda de Oro en Rusia; Ilkanato en Persia; Kanato de Chagatai en el corazón de la tierra de los mongoles y; la dinastía Yuan en China. Principalmente porque los unía el compromiso, el hecho que tuvieran lazos personales y su identidad mongólica. El autor también los ve como una gran multinacional, por las ganancias que los lideres extraían de estos territorios. Ringmar (2019) considera que en estos lugares hubo sistemas internacionales, porque, basado en sus marco conceptual, estos compartían una serie de instituciones, normas y reglas que los mantenían unidos.

No obstante, esto no queda tan claro cuando hace referencia a los lugares en donde no hubo sistemas internacionales. Ringmar (2019) determina que en África y América, aunque tenían claras formas de organización, no eran un sistema internacional. En el caso del primero, argumenta que los obstáculos geográficos y climáticos fue lo que impidió la construcción de estructuras políticas, o sea, Estados. A pesar de que este menciona: el Egipto faraónico, el Reino de Nubia, el Reino de Axum, de los Bereberes, de los Almorávides y los Almohades, el Imperio Mali y el Songhai, el Oba de Benín, la gente Akan y Yoruba, los estados del Savannah, el Reino Bunyoro, el Reino Buganda y la migración Bantú. Que han sido grandes imperios, con instituciones políticas que estaban conectadas, según el autor, por redes de intercambio internacional, 
pero no así en cuanto a sus relaciones políticas para determinarlos como regiones integradas

En cuanto a América, a menciona a los Mayas, los Incas, los Azteca y las comunidades ubicadas en el Norte de América, así como su historia, y explica que cada imperio siempre estuvo unido por un fuerte sentido de los valores políticos. En el caso de los Incas, el autor menciona al gran imperio de Tiwanaku en el primer milenio de la "Era Común", y ya cuando este se había establecido explica que era un Imperio centralizada y jerárquico, y que cuando empezó su expansión estos enviaban misiones diplomáticas para pedir alianzas y los beneficios que obtendrían al convertirse en sus súbditos. Así también, Ringmar (2019), determina que los Maya y los Aztecas crearon grandes federaciones que incluían una gran cantidad de grupos étnicos. Según Ringmar (2019) los Mayas no crearon un Estado centralizado, su imperio consistía en una federación de ciudades que incluían Palenque, Calakmul, Caracol, Mayapan y Tikal. Por último, cuando menciona a los Aztecas, este menciona al Imperio Tolteca, que duró 500 años, y con la llegada de los mexicas se da la alianza entre Tenochtitlán, Texcoco y Tlacopán, que formaron esta triple alianza para construir el Imperio Azteca. Sin embargo, Ringmar (2019) considera que no hubo un sistema internacional porque estos grupos no se conectaron nunca como uno.

A modo de epílogo, este es un texto de fácil comprensión para las personas lectoras, y tiene gran importancia la explicación que realiza sobre la historia de estos otros lugares que han quedado marginados y reducidos por la expansión de Europa. Se destaca que lo hace de una forma no exhaustiva, como una introducción a la historia, lo que convierte al libro en una lectura ligera para la cantidad de información que considera. Sin embargo, se debe señalar que el autor, aunque hace recomendaciones de lecturas, no cita investigaciones de otros autores que hagan más robustos sus argumentos.

El autor logra lo que pretende, una revisión histórica para determinar los sistemas internacionales previos al europeo, sin embargo, en cuanto al estudio comparativo, se queda corto en su profundización, porque, aunque intenta conectar las historias que van sucediendo al mismo tiempo, no lo se hace del todo. Además, es necesario que la idea sea transversal, porque después de la explicación histórica de los lugares, en la parte conclusiva no se dilucida completamente por qué es o no es un sistema internacional. Se considera que, al tomar una idea muy occidental, como es el concepto de Estado, para determinar si es o no un sistema internacional, se vuelve reduccionista determinar un sistema internacional. 
LOS SISTEMAS INTERNACIONALES NO EUROPEOS. RESEÑA DEL LIBRO:

RINGMAR, E. (2019). HISTORY OF INTERNATIONAL RELATIONS: A

NON-EUROPEAN PERSPECTIVE. OPEN BOOK PUBLISHERS.

\section{Referencias}

Ringmar, E. (2019). History of International Relations: A Non-European Perspective. Open Book Publishers. https://doi.org/10.11647/OBP.0074 
